\title{
A-unital Operations and Quantum Conditional Entropy
}

\author{
Mahathi Vempati ${ }^{1,2}$, Saumya Shah ${ }^{3}$, Nirman Ganguly ${ }^{4}$, and Indranil Chakrabarty ${ }^{1,5}$ \\ ${ }^{1}$ Centre for Quantum Science and Technology, \\ International Institute of Information Technology-Hyderabad, Gachibowli, Telangana-500032, India. \\ ${ }^{2}$ Center for Computational Natural Sciences and Bioinformatics, \\ International Institute of Information Technology-Hyderabad, Gachibowli, Telangana-500032, India. \\ ${ }^{3}$ Department of Physics, \\ Indian Institute of Technology Guwahati, Guwahati-781039, Assam, India. \\ ${ }^{4}$ Department of Mathematics, \\ Birla Institute of Technology and Science Pilani, Hyderabad Campus, Telangana-500078, India. \\ ${ }^{5}$ Center for Security, Theory and Algorithmic Research, \\ International Institute of Information Technology-Hyderabad, Gachibowli, Telangana-500032, India.
}

\begin{abstract}
Negative quantum conditional entropy states are key ingredients for information theoretic tasks such as superdense coding, state merging and one-way entanglement distillation. In this work, we ask: how does one detect if a channel is useful in preparing negative conditional entropy states? We answer this question by introducing the class of A-unital channels, which we show are the largest class of conditional entropy non-decreasing channels. We also prove that A-unital channels are precisely the completely free operations for the class of states with non-negative conditional entropy. Furthermore, we study the relationship between A-unital channels and other classes of channels pertinent to the resource theory of entanglement. We then prove similar results for ACVENN: a previously defined, relevant class of states and also relate the maximum and minimum conditional entropy of a state with its von Neumann entropy.

The definition of A-unital channels naturally lends itself to a procedure for determining membership of channels in this class. Thus, our work is valuable for the detection of resourceful channels in the context of conditional entropy.
\end{abstract}

\section{Introduction}

The myriad information-theoretic applications of entanglement [1] have elevated it from being merely an interesting physical phenomenon to that of a resource. In the resourcetheoretic study of entanglement, the separable states (quantum states that can be written as convex combinations of pure product states) are called the free states, and those outside this set, the entangled states, are known as the resource states [2]. The resource theory also

\footnotetext{
Mahathi Vempati: mahathi.vempati@research.iiit.ac.in

Saumya Shah: shah18@iitg.ac.in

Nirman Ganguly: nirmanganguly@gmail.com

Indranil Chakrabarty: indranil.chakrabarty@iiit.ac.in
} 
defines various classes of free operations, that is, quantum operations that cannot convert a free state to a resourceful one. Local Operations and Classical Communication (LOCC) is a physically defined class of free operations [1-3], characterized by the practical difficulty of communicating quantum information. A superset of LOCC is the class of separable channels $[2,4]$, which has a more appealing mathematical structure than LOCC and is therefore used to prove properties of LOCC itself [4, 5]. Entanglement-breaking channels form another important class of channels. These channels destroy all entanglement between a system and its environment [6].

Once these classes of channels have been defined, it is important to be able to decide whether or not a given channel belongs to any of these free classes. The quantum channel detection problem asks: given a channel and a well-defined class of channels, does the channel belong to the class [7-10]? The channel itself maybe provided as a blackbox, as in a laboratory setting, where it can be characterized by measuring its output on various input states, or as a full description (for instance, via its Kraus decomposition or Choi matrix). Unfortunately, for some classes defined with respect to entanglement, this problem is notoriously difficult. For example, it is currently unknown how to decide whether a given channel belongs to LOCC [2], and the problem of membership of a channel in the entanglement-breaking class is NP Hard [11]. There have been witness-based probabilistic approaches to deal with this problem, which are useful when some apriori information about a channel is known [7].

The interest in defining free operations for entanglement and studying mechanisms for detection of these operations is in part due to the resourcefulness of entanglement. While entanglement is certainly important in the characterization of quantum states for information-theoretic tasks, there are several phenomena for which merely possessing entanglement is not a sufficient condition to determine usefulness of a quantum state. Often, a state needs to have other properties beyond being entangled in order to be useful. One such property is the negativity of the quantum conditional entropy (also known as the conditional von Neumann entropy) of a quantum state [12]. The quantum conditional entropy (henceforth referred to as conditional entropy where there is no ambiguity) of a quantum state $\rho_{A B}$ is defined as

$$
S_{A \mid B}\left(\rho_{A B}\right)=S\left(\rho_{A B}\right)-S\left(\rho_{B}\right),
$$

where $S(\rho)$ is the von Neumann entropy (henceforth referred to as entropy where there is no ambiguity) of the state $\rho$, given by $S(\rho)=-\operatorname{Tr}(\rho \log \rho)$. All logarithms are taken to the base 2. Conditional entropy is used to define the information-theoretic quantities coherent information [13], which is just the negative of conditional entropy, and quantum discord [14-16], which is a measure of non-classical correlations in a quantum state. While all negative conditional entropy states are entangled, not all entangled states possess negative conditional entropy. Negative conditional entropy states are important for the informationtheoretic primitive quantum state merging [17, 18], and provide quantum advantage in superdense coding [19-21]. Negativity of conditional entropy is a necessary feature for the one-way distillation of entanglement [22]. It is important in maximizing the rates of distributed private randomness distillation [23], as well as in the reduction of uncertainty in predicting the outcomes of two incompatible measurements [24]. Previously, the set of states that are not useful for any of the above tasks, that is, the set of states possessing non-negative conditional entropy (we refer to this set as $\mathcal{F}^{c}(A \mid B)$ ) was characterized as convex and compact $[25,26] .{ }^{1}$ The set of states for which conditional entropy remains non-

\footnotetext{
${ }^{1}$ This is proven for the the case where $\operatorname{dim} A=\operatorname{dim} B=d[25]$, but a similar argument can be used to extend this proof to the case where $\operatorname{dim} A \neq \operatorname{dim} B$.
} 
negative even under the application of global unitary channels (referred to as $\mathcal{F}^{a c}(A \mid B)$ in this work) was also similarly characterized [27].

In this work, taking $\mathcal{F}^{c}(A \mid B)$ to be a set of free states, we ask: what is the class of free operations for $\mathcal{F}^{c}(A \mid B)$, and what are its properties? How does this class of operations relate to operations defined in the context of entanglement: separable channels and entanglement-breaking channels? How does one detect if a given channel belongs to the class of free operations for $\mathcal{F}^{c}(A \mid B)$ ? These questions are important in the context of preparation of quantum states for the aforementioned tasks, in order to determine if an operation one has access to is capable of generating resource.

This paper is organized as follows: In Section 2, we introduce the notation, and recap the relevant preliminaries. In Section 3, we define a class of free operations $\operatorname{NCVE}(A \mid B)$, the largest class of conditional entropy non-decreasing channels. We prove that this class is exactly equal to $\mathcal{O}_{\text {cmax }}^{c}(A \mid B)$, the largest class of completely free channels for $\mathcal{F}^{c}(A \mid B)$, and is elegantly characterized by a property we call $A$-unitality. We discuss properties of $A$-unital channels in Section 4. In Section 5, we discuss the relation between $A$-unital channels, separable channels and entanglement-breaking channels. While the set of separable states and $\mathcal{F}^{c}(A \mid B)$ share a subset-superset relationship, we show that surprisingly, this relationship does not hold for $A$-unital channels and separable channels-there exist $A$-unital channels that are not separable, separable channels that are not $A$-unital, as well as channels in the intersection. In Section 6, we extend the class $\mathcal{F}^{a c}(A \mid B)$, previously characterized only for the $2 \otimes 2$ case to the $d \otimes d$ case, and prove that unital channels are the largest class of completely free channels for $\mathcal{F}^{a c}(A \mid B)$. Since both entropy and conditional entropy are heavily discussed in this manuscript, in Section 7 , we provide upper and lower bounds for the conditional entropy of a state for a given entropy. We conclude in Section 8 with some concrete open questions that emerge from our work.

\section{Preliminaries}

In this section, we review relevant definitions and concepts from literature. $\mathcal{H}^{A}$ denotes a finite dimensional Hilbert space for physical system $A$. The dimension of the space $\mathcal{H}^{A}$ is given by $d_{A}$. The set of bounded linear operators on the Hilbert space $\mathcal{H}^{A}$ is denoted by $\mathcal{B}(A)$. The set of bounded linear operators over Hilbert space $\mathcal{H}^{A} \otimes \mathcal{H}^{B}$ is $\mathcal{B}(A B)$. The set of bounded linear operators that are Hermitian, positive semi-definite and have unit trace, that is, the density matrices (or quantum states) over $\mathcal{H}^{A}$ is denoted by $\mathcal{D}(A)$. A channel $\mathcal{N}: \mathcal{D}(A) \rightarrow \mathcal{D}(B)$ is a linear map from $\mathcal{D}(A)$ to $\mathcal{D}(B)$ that is completely positive and trace preserving (CPTP).

A quantum state $\rho_{A B}$ is called separable if it can be written as a convex combination of pure product states. That is, $\rho_{A B}$ is separable if $\rho_{A B}=\sum_{i} p_{i}\left|\alpha_{i}\right\rangle\left\langle\alpha_{i}\right|$ where $p_{i} \in[0,1]$, $\sum_{i} p_{i}=1$ and $\left|\alpha_{i}\right\rangle=\left|\phi_{i}\right\rangle_{A} \otimes\left|\psi_{i}\right\rangle_{B}$ for some $\left|\phi_{i}\right\rangle \in \mathcal{H}^{A}$ and $\left|\psi_{i}\right\rangle \in \mathcal{H}^{B}$. Separable states are the free states in the resource theory of entanglement. We denote the set of separable states in $\mathcal{D}(A B)$ by $\mathcal{F}^{s}(A \mid B)$. The set of states $\rho_{A B} \in \mathcal{D}(A B)$ for which $S_{A \mid B}\left(\rho_{A B}\right) \geq 0$, that is, the set of states possessing non-negative conditional entropy (Eq. 1) is denoted by $\mathcal{F}^{c}(A \mid B)$.

Free and completely free channels for a given set of free states were defined in [2]. We define these here for $\mathcal{F}^{c}(A \mid B)$.

Definition 1. A channel $\mathcal{N}_{A B \rightarrow C D}: \mathcal{D}(A B) \rightarrow \mathcal{D}(C D)$ is free for $\mathcal{F}^{c}(A \mid B)$ if for all $\rho \in \mathcal{F}^{c}(A \mid B)$, we have $\mathcal{N}_{A B \rightarrow C D}(\rho) \in \mathcal{F}^{c}(C \mid D)$. The class of all such free channels mapping $\mathcal{D}(A B)$ to $\mathcal{D}(C D)$ is denoted by $\mathcal{O}_{\text {max }}^{c}(A|B \rightarrow C| D)$. When the input and output spaces are the same, the class is denoted by $\mathcal{O}_{\text {max }}^{c}(A \mid B) \equiv \mathcal{O}_{\text {max }}^{c}(A|B \rightarrow A| B)$. 
Definition 2. A channel $\mathcal{N}_{A B \rightarrow C D}: \mathcal{D}(A B) \rightarrow \mathcal{D}(C D)$ is completely free for $\mathcal{F}^{c}(A \mid B)$ if for all finite dimensional Hilbert spaces $\mathcal{H}^{A^{\prime}}$ and $\mathcal{H}^{B^{\prime}}$ and $\rho \in \mathcal{F}^{c}\left(A^{\prime} A \mid B^{\prime} B\right)$, we have $\left(\operatorname{id}_{A^{\prime} B^{\prime}} \otimes \mathcal{N}_{A B \rightarrow C D}\right)(\rho) \in \mathcal{F}^{c}\left(A^{\prime} C \mid B^{\prime} D\right)$. The class of all such completely free channels mapping $\mathcal{D}(A B)$ to $\mathcal{D}(C D)$ is denoted by $\mathcal{O}_{\text {cmax }}^{c}(A|B \rightarrow C| D)$. When the input and output spaces are the same, the class is denoted by $\mathcal{O}_{\text {cmax }}^{c}(A \mid B) \equiv \mathcal{O}_{\text {cmax }}^{c}(A|B \rightarrow A| B)$.

The free and completely free channels for $\mathcal{F}^{s}(A \mid B)$, denoted by $\mathcal{O}_{\text {max }}^{s}(A|B \rightarrow C| D)$ and $\mathcal{O}_{\text {cmax }}^{s}(A|B \rightarrow C| D)$ respectively are defined analogously, replacing $\mathcal{F}^{c}(A \mid B)$ in the above definitions by $\mathcal{F}^{s}(A \mid B)$.

Separable channels [4] form an important class of channels in the entanglement resource theory. We define these channels here:

Definition 3. A channel $N: \mathcal{D}(A B) \rightarrow \mathcal{D}(C D)$ is called separable if and only if its action on a state $\rho \in \mathcal{D}(A B)$ can be expressed as

$$
\mathcal{N}(\rho)=\sum_{i=1}^{n}\left(K_{A}^{i} \otimes L_{B}^{i}\right) \rho\left(K_{A}^{i} \otimes L_{B}^{i}\right)^{\dagger}
$$

for some integer $n \geq 1$ and linear maps $K_{A}^{i}: \mathcal{H}^{A} \rightarrow \mathcal{H}^{C}$ and $L_{B}^{i}: \mathcal{H}^{B} \rightarrow \mathcal{H}^{D}$ such that $\sum_{i=1}^{n}\left(K_{A}^{i} \otimes L_{B}^{i}\right)^{\dagger}\left(K_{A}^{i} \otimes L_{B}^{i}\right)=I_{A B}$. The class of all separable channels mapping $\mathcal{D}(A B)$ to $\mathcal{D}(C D)$ is denoted by $\operatorname{SEP}(A|B \rightarrow C| D)$. When the input and output spaces are the same, the class is denoted by $\operatorname{SEP}(A \mid B) \equiv \operatorname{SEP}(A|B \rightarrow A| B)$.

It turns out that $\operatorname{SEP}(A \mid B)$ is exactly equal to $\mathcal{O}_{\text {cmax }}^{s}(A \mid B)[2]$. That is, the class of separable channels form the largest class of completely free channels for $\mathcal{F}^{s}(A \mid B)$.

Entanglement-breaking channels constitute another relevant class of channels [13]:

Definition 4. A channel $\mathcal{N}: \mathcal{D}(A) \rightarrow \mathcal{D}(B)$ is called entanglement-breaking if the quantum state $\left(\operatorname{id}_{R} \otimes \mathcal{N}\right)\left(\rho_{R A}\right)$ is separable for all $\rho_{R A}$ where $R$ is a reference system of arbitrary dimension. The class of all entanglement-breaking channels mapping $\mathcal{D}(A)$ to $\mathcal{D}(B)$ is denoted by $\mathrm{EB}(A \rightarrow B)$. When the input and output spaces are the same, the class is denoted by $\mathrm{EB}(A) \equiv \mathrm{EB}(A \rightarrow A)$.

Unital channels play an important role in the context of entropy, as the class of unital channels mapping $\mathcal{D}(A)$ to itself are exactly the ones that do not decrease the entropy of any state $\rho \in \mathcal{D}(A)[13]$. We define them as

Definition 5. A channel $\mathcal{N}: \mathcal{D}(A) \rightarrow \mathcal{D}(A)$ is called unital if $\mathcal{N}\left(\frac{I_{A}}{d_{A}}\right)=\frac{I_{A}}{d_{A}}$. The class of all unital channels mapping $\mathcal{D}(A)$ to $\mathcal{D}(A)$ is denoted by $\operatorname{UNI}(A)$.

Having reviewed the definitions, we now characterize the class of completely free operations for $\mathcal{F}^{c}(A \mid B)$.

\section{Completely Free Operations for Conditional Entropy}

Since the negativity of conditional entropy implies usefulness for various tasks, a natural candidate for a class of operations that are free for $\mathcal{F}^{c}(A \mid B)$ is the class of channels that does not decrease the conditional entropy of any state. Conditional entropy non-decreasing channels are defined as follows:

Definition 6. A channel $\mathcal{N}_{A B \rightarrow C D}: \mathcal{D}(A B) \rightarrow \mathcal{D}(C D)$ is said to be conditional von Neumann entropy (CVE) non-decreasing, if for all $\rho \in \mathcal{D}(A B)$, we have $S_{C \mid D}\left(\mathcal{N}_{A B \rightarrow C D}(\rho)\right) \geq$ $S_{A \mid B}(\rho)$. The class of $C V E$ non-decreasing channels mapping $\mathcal{D}(A B)$ to $\mathcal{D}(C D)$ is denoted by $\operatorname{NCVE}(A|B \rightarrow C| D)$. When the input and output spaces are the same, the class is denoted by $\operatorname{NCVE}(A \mid B) \equiv \operatorname{NCVE}(A|B \rightarrow A| B)$. 


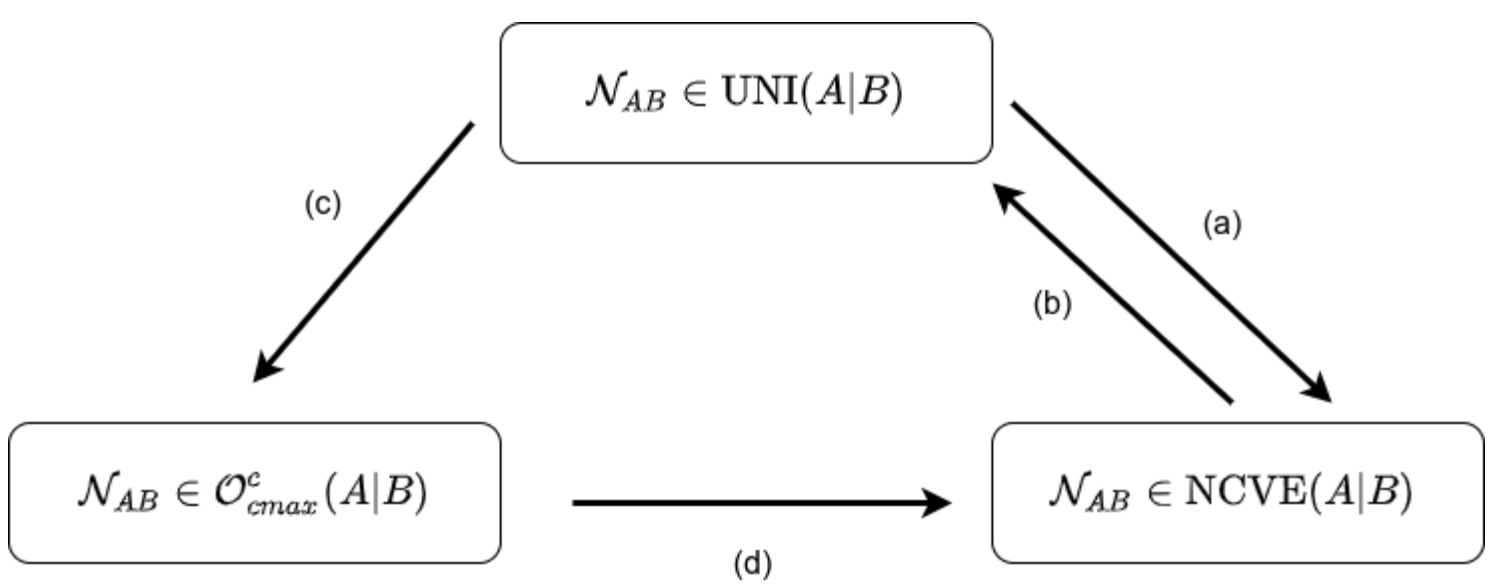

Figure 1: Equivalence of $\operatorname{UNI}(A \mid B), \operatorname{NCVE}(A \mid B)$ and $\mathcal{O}_{c m a x}^{c}(A \mid B)$.

It turns out that this class of channels is exactly equal to $\mathcal{O}_{c m a x}^{c}(A \mid B)$ which was introduced in Def. 2. We prove this equivalence by showing that both $\operatorname{NCVE}(A \mid B)$ and $\mathcal{O}_{\text {cmax }}^{c}(A \mid B)$ are in fact equal to a third class of operations, defined as follows:

Definition 7. A channel $\mathcal{N}: \mathcal{D}(A B) \rightarrow \mathcal{D}(A B)$ is called $A$-unital if for all $\rho_{B} \in \mathcal{D}(B)$, $\mathcal{N}$ satisfies

$$
\mathcal{N}\left(\frac{I_{A}}{d_{A}} \otimes \rho_{B}\right)=\frac{I_{A}}{d_{A}} \otimes \rho_{B}^{\prime}
$$

for some $\rho_{B}^{\prime} \in \mathcal{D}(B)$. We denote the set of $A$-unital channels on $\mathcal{D}(A B)$ as $\operatorname{UNI}(A \mid B)$. Likewise, the channel $\mathcal{N}$ is called $B$-unital if for all $\rho_{A} \in \mathcal{D}(A), \mathcal{N}$ satisfies

$$
\mathcal{N}\left(\rho_{A} \otimes \frac{I_{B}}{d_{B}}\right)=\rho_{A}^{\prime} \otimes \frac{I_{B}}{d_{B}}
$$

for some $\rho_{A}^{\prime} \in \mathcal{D}(A)$, and the set of $B$-unital channels on $\mathcal{D}(A B)$ is denoted as $\operatorname{UNI}(B \mid A)$.

Not only does the class of $A$-unital channels serve to show that both $\operatorname{NCVE}(A \mid B)$ and $\mathcal{O}_{\text {cmax }}^{c}(A \mid B)$ are equal, but its definition also makes this class amenable to detection, as discussed in Section 4.3. We now prove the equivalence of these three classes.

Theorem 1. The classes $\operatorname{UNI}(A \mid B), \operatorname{NCVE}(A \mid B)$ and $\mathcal{O}_{c m a x}^{c}(A \mid B)$ are all equal to each other.

Proof. The structure of the proof is depicted in Fig. 1. Let $\mathcal{N}: \mathcal{D}(A B) \rightarrow \mathcal{D}(A B)$ be a quantum channel. In Lemma 1 , we show that $\mathcal{N} \in \operatorname{UNI}(A \mid B) \Longrightarrow \mathcal{N} \in \operatorname{NCVE}(A \mid B)$, labelled as (a). In Lemma 2, we show that $\mathcal{N} \in \operatorname{NCVE}(A \mid B) \Longrightarrow \mathcal{N} \in \operatorname{UNI}(A \mid B)$, which is labelled as (b). In Lemma 3, we show that $\mathcal{N} \in \operatorname{UNI}(A \mid B) \Longrightarrow\left(\operatorname{id}_{A^{\prime} B^{\prime}} \otimes \mathcal{N}\right) \in$ $\mathrm{UNI}\left(A^{\prime} A \mid B^{\prime} B\right)$. We then use Lemma 1 and Lemma 3 to prove Lemma 4 , which is $\mathcal{N} \in$ $\mathrm{UNI}(A \mid B) \Longrightarrow \mathcal{N} \in \mathcal{O}_{c m a x}^{c}(A \mid B)$, labelled as (c) in the figure. Finally, we prove Lemma 5 , which is $\mathcal{N} \in \mathcal{O}_{\text {cmax }}^{c}(A \mid B) \Longrightarrow \mathcal{N} \in \operatorname{NCVE}(A \mid B)$, labelled as (d) in the figure. These lemmas establish the equivalence between the three classes.

Lemma 1. $\mathcal{N} \in \operatorname{UNI}(A \mid B) \Longrightarrow \mathcal{N} \in \operatorname{NCVE}(A \mid B)$. 
Proof. Let $\mathcal{N} \in \operatorname{UNI}(A \mid B)$. Thus, for all $\rho_{B} \in \mathcal{D}(B)$, we have $\mathcal{N}\left(\frac{I_{A}}{d_{A}} \otimes \rho_{B}\right)=\frac{I_{A}}{d_{A}} \otimes \rho_{B}^{\prime}$. To prove the Lemma, we require two results related to quantum relative entropy. The first result is a characterization of the conditional entropy in terms of relative entropy $[13,28]$ :

$$
\begin{aligned}
S_{A \mid B}\left(\rho_{A B}\right) & =\log d-S\left(\rho_{A B} \| \frac{I_{A}}{d_{A}} \otimes \rho_{B}\right) \\
& =\max _{\sigma_{B} \in \mathcal{D}(B)} \log d-S\left(\rho_{A B} \| \frac{I_{A}}{d_{A}} \otimes \sigma_{B}\right) .
\end{aligned}
$$

Next, we require the monotonicity of relative entropy, which is

$$
S(\mathcal{N}(\rho) \| N(\sigma)) \leq S(\rho \| \sigma) .
$$

From Eq. 5, for all $\rho \in \mathcal{D}(A B)$ and $\sigma_{B} \in \mathcal{D}(B)$, we have

$$
S_{A \mid B}(\mathcal{N}(\rho)) \geq \log d-S\left(\mathcal{N}(\rho) \| \frac{I_{A}}{d_{A}} \otimes \sigma_{B}\right) .
$$

In particular, we must have

$$
S_{A \mid B}(\mathcal{N}(\rho)) \geq \log d-S\left(\mathcal{N}(\rho) \| \frac{I_{A}}{d_{A}} \otimes \rho_{B}^{\prime}\right),
$$

where $\mathcal{N}\left(\frac{I_{A}}{d_{A}} \otimes \rho_{B}\right)=\frac{I_{A}}{d_{A}} \otimes \rho_{B}^{\prime}$. Therefore,

$$
S_{A \mid B}(\mathcal{N}(\rho)) \geq \log d-S\left(\mathcal{N}(\rho) \| \mathcal{N}\left(\frac{I_{A}}{d_{A}} \otimes \rho_{B}\right)\right) .
$$

From Eq. 6, we have

$$
\log d-S\left(\mathcal{N}(\rho) \| \mathcal{N}\left(\frac{I_{A}}{d_{A}} \otimes \rho_{B}\right)\right) \geq \log d-S\left(\rho \| \frac{I_{A}}{d_{A}} \otimes \rho_{B}\right)=S_{A \mid B}(\rho) .
$$

Thus, for all $\rho \in \mathcal{D}(A B)$, we have $S_{A \mid B}(\mathcal{N}(\rho)) \geq S_{A \mid B}(\rho)$, which implies that $\mathcal{N} \in$ $\operatorname{NCVE}(A \mid B)$.

Lemma 2. $\mathcal{N} \in \operatorname{NCVE}(A \mid B) \Longrightarrow \mathcal{N} \in \mathrm{UNI}(A \mid B)$.

Proof. For any $\rho_{A B} \in \mathcal{D}(A B)$, the maximum value of $S_{A \mid B}\left(\rho_{A B}\right)$ is $\log d_{A}$ and is attained if and only if $\rho_{A B}=\frac{I_{A}}{d_{A}} \otimes \sigma_{B}$ for some $\sigma_{B} \in \mathcal{D}(B)[13]$.

We use the above fact to prove the lemma by contradiction. Let $\mathcal{N} \in \operatorname{NCVE}(A \mid B)$. Assume that $\mathcal{N} \notin \operatorname{UNI}(A \mid B)$. Then, there exists some $\sigma_{B} \in \mathcal{D}(B)$ such that $\mathcal{N}\left(\frac{I_{A}}{d_{A}} \otimes \sigma_{B}\right) \neq \frac{I_{A}}{d_{A}} \otimes \sigma_{B}^{\prime}$ for all $\sigma_{B}^{\prime} \in \mathcal{D}(B)$. This implies that $S_{A \mid B}\left(\frac{I_{A}}{d_{A}} \otimes \sigma_{B}\right)=$ $\log d_{A}$, but $S_{A \mid B}\left(\mathcal{N}\left(\frac{I_{A}}{d_{A}} \otimes \sigma_{B}\right)\right)<\log d_{A}$. However, this is a contradiction since $\mathcal{N} \in \operatorname{NCVE}(A \mid B)$. Therefore, the assumption that $\mathcal{N} \notin \mathrm{UNI}(A \mid B)$ must be false, and we have $\mathcal{N} \in \operatorname{NCVE}(A \mid B) \Longrightarrow \mathcal{N} \in \operatorname{UNI}(A \mid B)$.

Lemma 3. $\mathcal{N} \in \operatorname{UNI}(A \mid B) \Longrightarrow\left(\operatorname{id}_{A^{\prime} B^{\prime}} \otimes \mathcal{N}\right) \in \operatorname{UNI}\left(A^{\prime} A \mid B^{\prime} B\right)$.

Proof. Let $\mathcal{N}_{A B} \in \mathrm{UNI}(A \mid B)$. Then, for all $\rho_{B} \in \mathcal{D}(B)$, we have $\mathcal{N}\left(I_{A} \otimes \rho_{B}\right)=I_{A} \otimes \rho_{B}^{\prime}$ for some $\rho_{B}^{\prime} \in \mathcal{D}(B)$. It is always possible to find a basis for any $\mathcal{D}(X)$, in which each basis element is a density matrix, that is, each basis element also belongs to $\mathcal{D}(X)$ [13]. 
Let $\left\{\beta_{i}^{\prime}\right\}_{i}$ be such a basis for $\mathcal{D}\left(B^{\prime}\right)$ and $\left\{\beta_{j}\right\}_{j}$ be such a basis for $\mathcal{D}(B)$. Thus, $\left\{\beta_{i}^{\prime} \otimes \beta_{j}\right\}_{i j}$ is a basis of density matrices for $\mathcal{D}\left(B^{\prime} B\right)$. We have,

$$
\begin{aligned}
\left(\operatorname{id}_{A^{\prime} B^{\prime}}\right. & \left.\otimes \mathcal{N}_{A B}\right)\left(\frac{I_{A^{\prime} A}}{d_{A^{\prime} A}} \otimes\left(\beta_{i}^{\prime} \otimes \beta_{j}\right)_{B^{\prime} B}\right) \\
& =\left(i_{A^{\prime} B^{\prime}} \otimes \mathcal{N}_{A B}\right)\left(\frac{I_{A^{\prime}}}{d_{A^{\prime}}} \otimes \frac{I_{A}}{d_{A}} \otimes \beta_{i B^{\prime}}^{\prime} \otimes \beta_{j B}\right) \\
& =\frac{I_{A^{\prime}}}{d_{A^{\prime}}} \otimes \beta_{i B^{\prime}}^{\prime} \otimes \mathcal{N}_{A B}\left(\frac{I_{A}}{d_{A}} \otimes \beta_{j B}\right) \\
& =\frac{I_{A^{\prime}}}{d_{A^{\prime}}} \otimes \beta_{i B^{\prime}}^{\prime} \otimes \frac{I_{A}}{d_{A}} \otimes \gamma_{j B} \\
& =\frac{I_{A^{\prime} A}}{d_{A^{\prime} A}} \otimes\left(\beta_{i}^{\prime} \otimes \gamma_{j}\right)_{B^{\prime} B} .
\end{aligned}
$$

Now, for all $\sigma \in \mathcal{D}\left(B^{\prime} B\right)$, we have

$$
\begin{aligned}
\left(\operatorname{id}_{A^{\prime} B^{\prime}} \otimes \mathcal{N}_{A B}\right)\left(\frac{I_{A^{\prime} A}}{d_{A^{\prime} A}} \otimes \sigma_{B^{\prime} B}\right) \\
=\left(\operatorname{id}_{A^{\prime} B^{\prime}} \otimes \mathcal{N}_{A B}\right)\left(\frac{I_{A^{\prime} A}}{d_{A^{\prime} A}} \otimes\left(\sum_{i j} b_{i j} \beta_{i}^{\prime} \otimes \beta_{j}\right)_{B^{\prime} B}\right) \\
=\sum_{i j} b_{i j}\left(i_{A^{\prime} B^{\prime}} \otimes \mathcal{N}_{A B}\right)\left(\frac{I_{A^{\prime} A}}{d_{A^{\prime} A}} \otimes\left(\beta_{i}^{\prime} \otimes \beta_{j}\right)_{B^{\prime} B}\right) \\
=\sum_{i j} b_{i j} \frac{I_{A^{\prime} A}}{d_{A^{\prime} A}} \otimes\left(\beta_{i}^{\prime} \otimes \gamma_{j}\right)_{B^{\prime} B} \\
=\frac{I_{A^{\prime} A}}{d_{A^{\prime} A}} \otimes \sum_{i j} b_{i j}\left(\beta_{i}^{\prime} \otimes \gamma_{j}\right)_{B^{\prime} B} .
\end{aligned}
$$

Thus, $\operatorname{id}_{A^{\prime} B^{\prime}} \otimes \mathcal{N}_{A B} \in \mathrm{UNI}\left(A^{\prime} A \mid B^{\prime} B\right)$.

Lemma 4. $\mathcal{N} \in \operatorname{UNI}(A \mid B) \Longrightarrow \mathcal{N} \in \mathcal{O}_{c m a x}^{c}(A \mid B)$.

Proof. Let $\mathcal{N}_{A B} \in \operatorname{UNI}(A \mid B)$. From Lemma 3, we have $\operatorname{id}_{A^{\prime} B^{\prime}} \otimes \mathcal{N}_{A B} \in \operatorname{UNI}\left(A^{\prime} A \mid B^{\prime} B\right)$. Hence, using Lemma 1, we have $\operatorname{id}_{A^{\prime} B^{\prime}} \otimes \mathcal{N}_{A B} \in \operatorname{NCVE}\left(A^{\prime} A \mid B^{\prime} B\right)$. Thus, for all $\rho \in \mathcal{F}^{c}\left(A^{\prime} A \mid B^{\prime} B\right)$, we have $S_{A \mid B}\left(\left(\operatorname{id}_{A^{\prime} B^{\prime}} \otimes N_{A B}\right)(\rho)\right) \geq S_{A \mid B}(\rho) \geq 0$, which implies $\left(\operatorname{id}_{A^{\prime} B^{\prime}} \otimes \mathcal{N}_{A B}\right)(\rho) \in \mathcal{F}^{c}\left(A^{\prime} A \mid B^{\prime} B\right)$. Now, using Definition 2, we have $\mathcal{N}_{A B} \in$ $\mathcal{O}_{\text {cmax }}^{c}(A \mid B)$.

Lemma 5. $\mathcal{N} \in \mathcal{O}_{\text {cmax }}^{c}(A \mid B) \Longrightarrow \mathcal{N} \in \operatorname{NCVE}(A \mid B)$.

Proof. In order to prove the lemma, we prove the contrapositive statement, that is $\mathcal{N}_{A B} \notin$ $\operatorname{NCVE}(A \mid B) \Longrightarrow \mathcal{N}_{A B} \notin \mathcal{O}_{c m a x}^{c}(A \mid B)$. Let $\mathcal{N}_{A B}: \mathcal{D}(A B) \rightarrow \mathcal{D}(A B)$ be a quantum channel that does not belong to $\operatorname{NCVE}(A \mid B)$. Thus there exists $\rho \in \mathcal{D}(A B)$ such that $S_{A \mid B}(\mathcal{N}(\rho))<S_{A \mid B}(\rho)$. Consider Hilbert spaces $\mathcal{H}^{A^{\prime}}$ and $\mathcal{H}^{B^{\prime}}$, with $d_{A^{\prime}}=d_{A}$ and $d_{B^{\prime}}=$ $d_{B}$. Now, we select a state $\sigma \in \mathcal{D}\left(A^{\prime} B^{\prime}\right)$ such that

$$
-S_{A \mid B}(\rho) \leq S_{A^{\prime} \mid B^{\prime}}(\sigma)<-S_{A \mid B}(\mathcal{N}(\rho)) .
$$

Note that because $-S_{A \mid B}(\rho)<-S_{A \mid B}(\mathcal{N}(\rho))$ and both these values lie in $\left[-\log d_{A}, \log d_{A}\right]=\left[-\log d_{A^{\prime}}, \log d_{A^{\prime}}\right]$, the state $\sigma$ always exists. 
Now, consider the state $\sigma_{A^{\prime} B^{\prime}} \otimes \rho_{A B} \in \mathcal{D}\left(A^{\prime} B^{\prime} A B\right)$. Using Eq. 13, we have $S_{A^{\prime} A \mid B^{\prime} B}\left(\sigma_{A^{\prime} B^{\prime}} \otimes \rho_{A B}\right)=S_{A^{\prime} \mid B^{\prime}}(\sigma)+S_{A \mid B}(\rho) \geq-S_{A \mid B}(\rho)+S_{A \mid B}(\rho)=0$. Thus, $\sigma_{A^{\prime} B^{\prime}} \otimes \rho_{A B} \in \mathcal{F}^{c}\left(A^{\prime} A \mid B^{\prime} B\right)$.

We now apply the operation $\operatorname{id}_{A^{\prime} B^{\prime}} \otimes \mathcal{N}_{A B}$ on the state $\sigma_{A^{\prime} B^{\prime}} \otimes \rho_{A B}$. Once again, using Eq. 13, we have $S_{A^{\prime} A \mid B^{\prime} B}\left(\operatorname{id}_{A^{\prime} B^{\prime}} \otimes \mathcal{N}_{A B}\left(\sigma_{A^{\prime} B^{\prime}} \otimes \rho_{A B}\right)\right)=S_{A^{\prime} A \mid B^{\prime} B}\left(\sigma_{A^{\prime} B^{\prime}} \otimes \mathcal{N}_{A B}\left(\rho_{A B}\right)\right)$ $=S_{A^{\prime} \mid B^{\prime}}\left(\sigma_{A B}\right)+S_{A \mid B}\left(\mathcal{N}_{A B}\left(\rho_{A B}\right)\right)<-S_{A \mid B}\left(\mathcal{N}_{A B}\left(\rho_{A B}\right)\right)+S_{A \mid B}\left(\mathcal{N}_{A B}\left(\rho_{A B}\right)\right)=0$. This implies that $\operatorname{id}_{A^{\prime} B^{\prime}} \otimes \mathcal{N}_{A B}\left(\sigma_{A^{\prime} B^{\prime}} \otimes \rho_{A B}\right) \notin \mathcal{F}^{c}\left(A^{\prime} A \mid B^{\prime} B\right)$. Therefore, the channel $\operatorname{id}_{A^{\prime} B^{\prime}} \otimes \mathcal{N}_{A B}$ takes a state from inside $\mathcal{F}^{c}\left(A^{\prime} A \mid B^{\prime} B\right)$ to outside, and using Def. 2, $\mathcal{N}_{A B} \notin \mathcal{O}_{\text {cmax }}^{c}(A \mid B)$.

Thus, Lemmas 1-5 prove all the implications depicted in Fig. 1, and Theorem 1 follows.

\section{Properties of A-Unital channels}

\subsection{Serial and Parallel Concatenation}

Since we have $\operatorname{UNI}(A \mid B)=\mathcal{O}_{c m a x}^{c}(A \mid B)$, it follows that serial concatenation of two $A$ unital channels is $A$-unital, and parallel concatenation of an $A^{\prime}$-unital channel and an $A$-unital channel is $A^{\prime} A$-unital [2]. However, this is also readily seen from the definition of $\mathrm{UNI}(A \mid B)$. Let $\mathcal{M}_{A B}, \mathcal{N}_{A B} \in \mathrm{UNI}(A \mid B)$. Then, we have

$$
\mathcal{M}_{A B} \circ \mathcal{N}_{A B}\left(\frac{I_{A}}{d_{A}} \otimes \rho_{B}\right)=\mathcal{M}_{A B}\left(\frac{I_{A}}{d_{A}} \otimes \sigma_{B}\right)=\frac{I_{A}}{d_{A}} \otimes \gamma_{B} .
$$

Thus, $\mathcal{M}_{A B} \circ \mathcal{N}_{A B} \in \operatorname{UNI}(A \mid B)$. Similarly, if $\mathcal{M}_{A^{\prime} B^{\prime}} \in \operatorname{UNI}\left(A^{\prime} \mid B^{\prime}\right)$, and $\mathcal{N}_{A B} \in \operatorname{UNI}(A \mid B)$, from Lemma 3, we have $\left(\operatorname{id}_{A^{\prime} B^{\prime}} \otimes \mathcal{N}_{A B}\right),\left(\mathcal{M}_{A^{\prime} B^{\prime}} \otimes \operatorname{id}_{A B}\right) \in \operatorname{UNI}\left(A^{\prime} A \mid B^{\prime} B\right)$. Then, from the closedness under serial concatenation above, it follows that

$$
\left(\operatorname{id}_{A^{\prime} B^{\prime}} \otimes \mathcal{N}_{A B}\right) \circ\left(\mathcal{M}_{A^{\prime} B^{\prime}} \otimes \mathrm{id}_{A B}\right)=\mathcal{M}_{A^{\prime} B^{\prime}} \otimes \mathcal{N}_{A B} \in \mathrm{UNI}\left(A^{\prime} A \mid B^{\prime} B\right) .
$$

\subsection{Convexity}

Theorem 2. A convex combination of A-unital channels is an A-unital channel.

Proof. Let $\mathcal{N}_{1}, \mathcal{N}_{2} \in \mathrm{UNI}(A \mid B)$. Then we have,

$$
\begin{aligned}
\left(p \mathcal{N}_{1}+(1-p) \mathcal{N}_{2}\right) & \left(\frac{I_{A}}{d_{A}} \otimes \beta_{B}\right) \\
& =p \mathcal{N}_{1}\left(\frac{I_{A}}{d_{A}} \otimes \beta_{B}\right)+(1-p) \mathcal{N}_{2}\left(\frac{I_{A}}{d_{A}} \otimes \beta_{B}\right) \\
& =p\left(\frac{I_{A}}{d_{A}} \otimes \gamma_{1 B}\right)+(1-p)\left(\frac{I_{A}}{d_{A}} \otimes \gamma_{2 B}\right) \\
& =\frac{I_{A}}{d_{A}} \otimes\left(p \gamma_{1}+(1-p) \gamma_{2}\right)_{B}
\end{aligned}
$$

Thus, $p \mathcal{N}_{1}+(1-p) \mathcal{N}_{2} \in \operatorname{UNI}(A \mid B)$.

\subsection{Detection}

The characterization of both conditional entropy non-decreasing channels and completely free channels for $\mathcal{F}^{c}(A \mid B)$ as $A$-unital channels makes apparent how these channels can be detected. For any $\mathcal{D}(B)$, it is always possible to find $d_{B}^{2}$ density matrices $\left\{\beta_{i}\right\}_{i}$ from $\mathcal{D}(B)$ that form a basis for for $\mathcal{D}(B)$ [13]. For some unknown channel $\mathcal{N}$ given as a blackbox, a necessary and sufficient condition for $A$-unitality is $\mathcal{N}\left(\frac{I_{A}}{d_{A}} \otimes \beta_{i B}\right)=\frac{I_{A}}{d_{B}} \otimes \beta_{i B}^{\prime}$ for all the $d_{B}^{2}$ states $\beta_{i}$ from the basis. This follows using the same proof technique as in Lemma 3. 


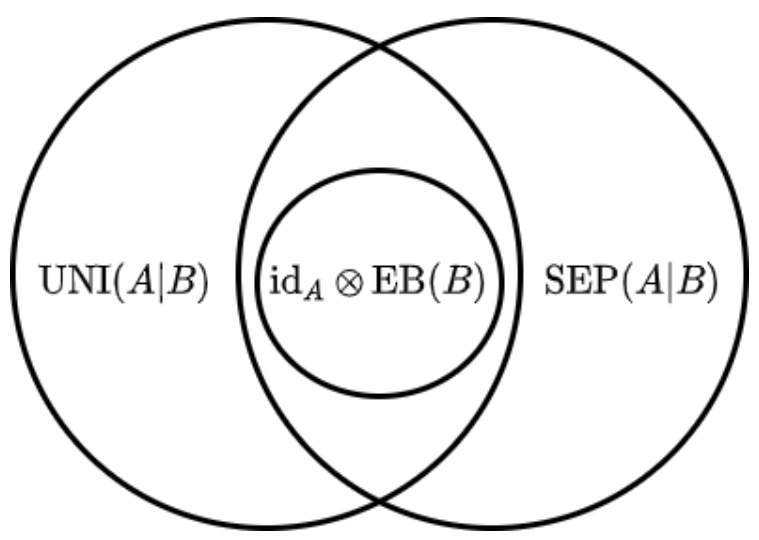

Figure 2: The relationship between $\operatorname{UNI}(A \mid B), \operatorname{SEP}(A \mid B)$ and $\operatorname{id}_{A} \otimes \operatorname{EB}(B)$ when $d_{A}=d_{B}$.

\section{Relationship between A-Unital Channels and Other Classes}

We now turn our attention to the relationship between $A$-unital channels and other classes. If we detect that a channel is $A$-unital (or not $A$-unital), can anything be said about the membership of the channel in $\operatorname{SEP}(A \mid B)$ ? It turns out that this is not the case, as there exist $A$-unital channels that are not separable, separable channels that are not $A$ unital, as well as channels in the intersection. We discuss this in the following subsections. We also discuss the relationship between entanglement breaking channels and $A$-unital channels. These relationships are depicted in Fig. 2. Throughout this section, we assume $d_{A}=d_{B}=d$.

\subsection{A-Unital Non-Separable Channels}

We prove the existence of channels that belong to $\operatorname{UNI}(A \mid B)$ but not $\operatorname{SEP}(A \mid B)$ by constructing such a channel. Let $\{|i\rangle\}_{i}$ be an orthonormal basis for $\mathcal{H}^{A}$, and $\{|j\rangle\}_{j}$ be an orthonormal basis for $\mathcal{H}^{B}$.

Definition 8. The Swap and Prepare channel, denoted by $\mathcal{P}_{A B}: \mathcal{D}(A B) \rightarrow \mathcal{D}(A B)$ is defined as the composition of the following channels:

- The Swap Channel $\left[S w_{A B}: \mathcal{D}(A B) \rightarrow \mathcal{D}(A B)\right]$. The unitary channel that swaps systems $A$ and $B$. Its Kraus decomposition contains just one Kraus operator which is the unitary matrix $U_{s} \equiv \sum_{i j}|j i\rangle\langle i j|$. We have

$$
S w_{A B}(\rho)=U_{s} \rho U_{s}^{\dagger} .
$$

- The Partial Trace Channel $\left[\operatorname{Tr}_{A}: \mathcal{D}(A B) \rightarrow \mathcal{D}(B)\right]$. The Kraus operators for this channel are $\left\{\left\langle\left. i\right|_{A} \otimes I_{B}\right\}_{i}\right.$. We have

$$
\operatorname{Tr}_{A}(\rho)=\sum_{i}\left(\left\langle\left. i\right|_{A} \otimes I_{B}\right)\left(\rho_{A B}\right)\left(|i\rangle_{A} \otimes I_{B}\right) .\right.
$$

- The Preparation of $\frac{I_{A}}{d}$ Channel $\left[\operatorname{Pr}_{A}: \mathcal{D}(B) \rightarrow \mathcal{D}(A B)\right]$. The Kraus operators for this channel are $\left\{\frac{1}{\sqrt{d}}|i\rangle_{A} \otimes I_{B}\right\}_{i}$. We have

$$
\begin{aligned}
\operatorname{Pr}_{A}\left(\rho_{B}\right) & =\sum_{i} \frac{1}{d}\left(|i\rangle_{A} \otimes I_{B}\right)\left(\rho_{B}\right)\left(\left\langle\left. i\right|_{A} \otimes I_{B}\right)\right. \\
& =\frac{I_{A}}{d} \otimes \rho_{B}
\end{aligned}
$$


Finally, we define the Swap and Prepare Channel:

$$
\mathcal{P}_{A B}\left(\rho_{A B}\right)=\left(\operatorname{Pr}_{A} \circ \operatorname{Tr}_{A} \circ S w_{A B}\right)\left(\rho_{A B}\right)
$$

Theorem 3. There exist A-unital non-separable channels.

Proof. In Lemma 6, we show that the channel $\mathcal{P}_{A B} \in \operatorname{UNI}(A \mid B)$, and in Lemma 7 , we show that $\mathcal{P}_{A B} \notin \operatorname{SEP}(A \mid B)$.

Lemma 6. $\mathcal{P}_{A B} \in \mathrm{UNI}(A \mid B)$.

Proof. Let $\sigma_{B} \in \mathcal{B}(B)$. We have

$$
\begin{aligned}
\mathcal{P}_{A B}\left(\frac{I_{A}}{d} \otimes \sigma_{B}\right) & =\operatorname{Pr}_{A} \circ \operatorname{Tr}_{A} \circ S w_{A B}\left(\frac{I_{A}}{d} \otimes \sigma_{B}\right)=\operatorname{Pr}_{A} \circ \operatorname{Tr}_{A}\left(\sigma_{A} \otimes \frac{I_{B}}{d}\right) \\
& =\operatorname{Pr}_{A}\left(\frac{I_{B}}{d}\right)=\frac{I_{A}}{d} \otimes \frac{I_{B}}{d} .
\end{aligned}
$$

Thus, using Def. 7, $\mathcal{P}_{A B} \in \mathrm{UNI}(A \mid B)$.

Lemma 7. $\mathcal{P}_{A B} \notin \operatorname{SEP}(A \mid B)$.

Proof. From the discussion following Def. 3, we have that $\operatorname{SEP}(A \mid B)=\mathcal{O}_{\text {cmax }}^{s}(A \mid B)$. Thus, it is sufficient to prove that $\mathcal{P}_{A B} \notin \mathcal{O}_{\text {cmax }}^{s}(A \mid B)$. Consider Hilbert spaces $\mathcal{H}^{A^{\prime}}$ and $\mathcal{H}^{B^{\prime}}$ where $d_{A^{\prime}}$ and $d_{B^{\prime}}$ are also equal to $d$. Take the state $\chi \in \mathcal{D}\left(A^{\prime} A B^{\prime} B\right)$ defined as $\chi \equiv$ $\left.\left|\phi^{+}\right\rangle \phi^{+}\right|_{A^{\prime} A} \otimes|00\rangle\left\langle\left. 00\right|_{B^{\prime} B}\right.$, where $\left.\mid \phi^{+}\right\rangle\left\langle\left.\phi^{+}\right|_{A^{\prime} A}\right.$ is the entangled state $\left.\frac{1}{d} \sum_{i j} \mid i\right\rangle\left\langle\left. j\right|_{A^{\prime}} \otimes \mid i\right\rangle\left\langle\left. j\right|_{A}\right.$. The state $\chi$ is a product state across the $A^{\prime} A \mid B^{\prime} B$ cut, and hence $\chi \in \mathcal{F}^{s}\left(A^{\prime} A \mid B^{\prime} B\right)$. We now apply $\operatorname{id}_{A^{\prime} B^{\prime}} \otimes \mathcal{P}_{A B}$ to the state $\chi$.

$$
\begin{aligned}
\chi^{\prime} & =\left(\operatorname{id}_{A^{\prime} B^{\prime}} \otimes \mathcal{P}_{A B}\right)(\chi) \\
& =\left(\operatorname{id}_{A^{\prime} B^{\prime}} \otimes \mathcal{P}_{A B}\right)\left(\frac{1}{d} \sum_{i j}|i\rangle\left\langle\left. j\right|_{A^{\prime}} \otimes \mid i\right\rangle\left\langle\left. j\right|_{A} \otimes \mid 0\right\rangle\left\langle\left. 0\right|_{B^{\prime}} \otimes \mid 0\right\rangle\left\langle\left. 0\right|_{B}\right)\right. \\
& =\left(\operatorname{id}_{A^{\prime} B^{\prime}} \otimes \operatorname{Pr}_{A} \circ \operatorname{Tr}_{A} \circ S w_{A B}\right)\left(\frac{1}{d} \sum_{i j}|i\rangle\left\langle\left. j\right|_{A^{\prime}} \otimes \mid i\right\rangle\left\langle\left. j\right|_{A} \otimes \mid 0\right\rangle\left\langle\left. 0\right|_{B^{\prime}} \otimes \mid 0\right\rangle\left\langle\left. 0\right|_{B}\right)\right. \\
& =\left(\operatorname { i d } _ { A ^ { \prime } B ^ { \prime } } ( \frac { 1 } { d } \sum _ { i j } | i \rangle \langle j | _ { A ^ { \prime } } \otimes | 0 \rangle \langle 0 | _ { B ^ { \prime } } ) ) \otimes \left(\left(P r_{A} \circ \operatorname{Tr}_{A} \circ S w_{A B}\right)\left(|i\rangle\left\langle\left. j\right|_{A} \otimes \mid 0\right\rangle\left\langle\left. 0\right|_{B}\right)\right)\right.\right. \\
& =\left(\frac { 1 } { d } \sum _ { i j } | i \rangle \langle j | _ { A ^ { \prime } } \otimes | 0 \rangle \langle 0 | _ { B ^ { \prime } } ) \otimes \left(\left(P r_{A} \circ \operatorname{Tr}_{A}\right)\left(|0\rangle\left\langle\left. 0\right|_{A} \otimes \mid i\right\rangle\left\langle\left. j\right|_{B}\right)\right)\right.\right. \\
& =\left(\frac { 1 } { d } \sum _ { i j } | i \rangle \langle j | _ { A ^ { \prime } } \otimes | 0 \rangle \langle 0 | _ { B ^ { \prime } } ) \otimes \left(P r_{A}\left(|i\rangle\left\langle\left. j\right|_{B}\right)\right)\right.\right. \\
& =\frac{1}{d} \sum_{i j}|i\rangle\left\langle\left. j\right|_{A^{\prime}} \otimes \mid 0\right\rangle\left\langle\left. 0\right|_{B^{\prime}} \otimes \frac{I_{A}}{d} \otimes \mid i\right\rangle\left\langle\left. j\right|_{B}\right. \\
& =\left|\phi^{+}\right\rangle\left\langle\left.\phi^{+}\right|_{A^{\prime} B} \otimes \frac{I_{A}}{d} \otimes \mid 0\right\rangle\left\langle\left. 0\right|_{B^{\prime}} .\right.
\end{aligned}
$$

Observe that $\chi^{\prime}$ is entangled across the $A^{\prime} A \mid B^{\prime} B$ cut. Thus, $\chi^{\prime} \notin \mathcal{F}^{s}\left(A^{\prime} A \mid B^{\prime} B\right)$. From the definition of $\mathcal{O}_{\text {cmax }}^{s}(A \mid B)$, this implies that $\mathcal{P}_{A B} \notin \mathcal{O}_{\text {cmax }}^{s}(A \mid B)$, hence $\mathcal{P}_{A B} \notin \operatorname{SEP}(A \mid B)$. The action of $\mathcal{P}_{A B}$ is depicted in Fig. 3.

From Lemmas 6 and 7 , we have that $\mathcal{P}_{A B} \in \operatorname{UNI}(A \mid B)$ but $\mathcal{P}_{A B} \notin \operatorname{SEP}(A \mid B)$, and thus, Theorem 3 follows. 
(1)
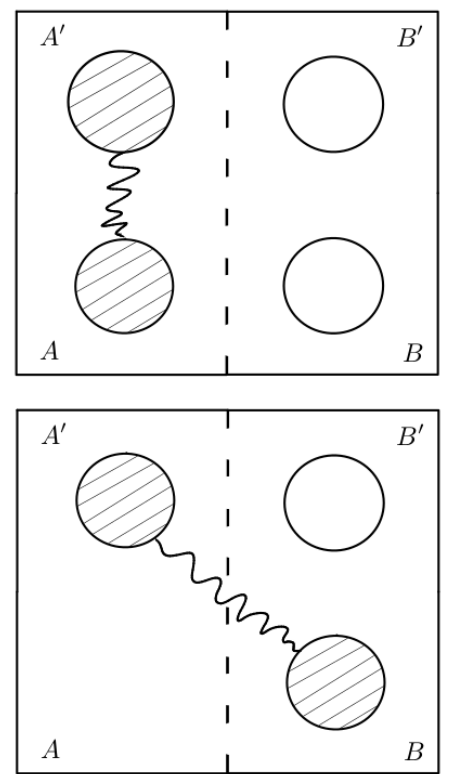

(3)
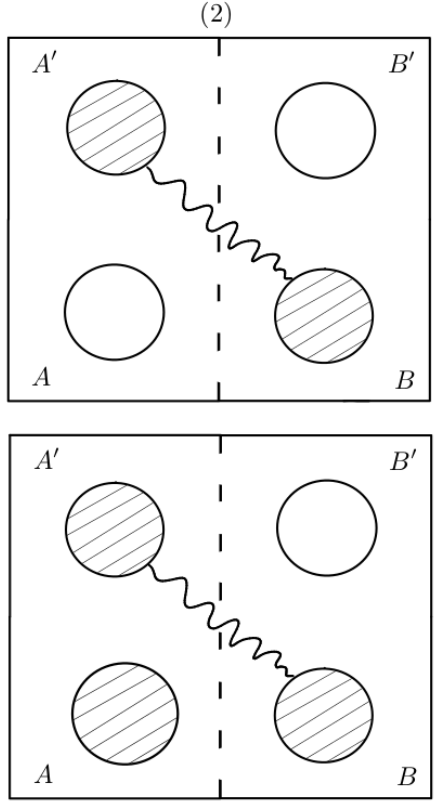

(4)

Figure 3: Demonstration of $\mathcal{P}_{A B}$ acting on $\chi$. Subfigure (1) depicts the state $\chi$, which is separable across the $A^{\prime} A \mid B^{\prime} B$ cut. Upon applying $\operatorname{id}_{A^{\prime} B^{\prime}} \otimes S w_{A B}$, the states of subsystems $A$ and $B$ are swapped, as shown in subfigure (2). Application of $\operatorname{Tr}_{A}$ is depicted in subfigure (3), and finally, $\operatorname{Pr}_{A}$ prepares the state $\frac{I_{A}}{d}$ and this is depicted in subfigure (4). Thus, $\mathcal{P}_{A B}$ is $A$-unital, but not separable, as the channel generates entanglement across the $A^{\prime} A \mid B^{\prime} B$ cut.

\subsection{Non-A-Unital Separable Channels}

Examples of channels that are non-A-unital but separable are replacement channels [2] $R$ which trace out a given state and prepare a product state $\rho_{A} \otimes \rho_{B}$ where $\rho_{A} \neq \frac{I_{A}}{d}$. Since for all $\sigma_{B} \in \mathcal{D}(B)$, we have $R\left(\frac{I_{A}}{d} \otimes \sigma_{B}\right) \neq \frac{I_{A}}{d} \otimes \gamma_{B}$ for some $\gamma \in \mathcal{D}(B), R$ is not an $A$-unital channel. However, since both tracing out as well as preparation of a product state are separable channels, $R$ is a separable channel. A specific example of a channel which prepares the state $|00\rangle\left\langle\left. 00\right|_{A B}\right.$ is

$$
R_{0}\left(\rho_{A B}\right)=\sum_{i j}\left(| 0 \rangle \langle i | _ { A } \otimes | 0 \rangle \langle j | _ { B } ) ( \rho _ { A B } ) \left(|i\rangle\left\langle\left. 0\right|_{A} \otimes \mid j\right\rangle\left\langle\left. 0\right|_{B}\right)\right.\right.
$$

where $\{|i\rangle\}_{i}$ and $\{|j\rangle\}_{j}$ are orthonormal bases for $\mathcal{H}^{A}$ and $\mathcal{H}^{B}$ respectively.

\subsection{A-Unital Separable Channels}

A trivial example of a channel that is both $A$-unital and separable is the identity channel. A more non-trivial example is any channel of the form

$$
C_{A B}(\rho)=\sum_{i}\left(K_{A}^{i} \otimes L_{B}^{i}\right) \rho\left(K_{A}^{i} \otimes L_{B}^{i}\right)^{\dagger}
$$


where $\sum_{i}\left(K_{A}^{i} \otimes L_{B}^{i}\right)^{\dagger}\left(K_{A}^{i} \otimes L_{B}^{i}\right)=I_{A B}$ and each $K_{A}^{i}$ is a unitary channel. $C_{A B}$ is separable by definition. It is also $A$-unital as shown below. Let $\sigma \in \mathcal{D}(B)$. We have

$$
\begin{aligned}
C_{A B}\left(\frac{I_{A}}{d} \otimes \sigma_{B}\right) & =\sum_{i}\left(K_{A}^{i} \otimes L_{B}^{i}\right)\left(\frac{I_{A}}{d} \otimes \sigma_{B}\right)\left(K_{A}^{i} \otimes L_{B}^{i}\right)^{\dagger} \\
& =\sum_{i} \frac{1}{d} K_{A}^{i} I_{A} K_{A}^{i \dagger} \otimes L_{B}^{i} \sigma_{B} L_{B}^{i \dagger}=\frac{I_{A}}{d} \otimes \sum_{i} L_{B}^{i} \sigma_{B} L_{B}^{i \dagger} .
\end{aligned}
$$

\subsection{A-Unital and Entanglement-Breaking Channels}

Both $\operatorname{UNI}(A \mid B)$ and $\operatorname{SEP}(A \mid B)$ are concerned with correlations across the $A \mid B$ split. Thus, we relate both these classes with channels of the form $\operatorname{id}_{A} \otimes \Phi_{B}$ where $\Phi_{B} \in \operatorname{EB}(B)$, that is $\Phi_{B}$ is an entanglement-breaking channel (Def. 4), as they destroy all entanglement across the $A \mid B$ split. These channels are $A$-unital, as $\left(\operatorname{id}_{A} \otimes \Phi_{B}\right)\left(\frac{I_{A}}{d} \otimes \sigma_{B}\right)=\frac{I_{A}}{d} \otimes \Phi(\sigma)_{B}$ for all $\sigma_{B} \in \mathcal{D}(B)$, as well as separable, as the Kraus operators for the channel are $\left\{I_{A} \otimes K_{B}^{i}\right\}_{i}$ where $\left\{K_{B}^{i}\right\}_{i}$ are the Kraus operators for $\Phi_{B}$. This is depicted in Figure 2. On the contrary, channels of the form $\Phi_{A} \otimes \mathrm{id}_{B}$ where $\Phi_{A} \in \operatorname{EB}(A)$ are not necessarily $A$-unital, although they are separable. As an example, consider the following classical-quantum channel $\mathcal{M}_{A}: \mathcal{D}(A) \rightarrow \mathcal{D}(A)$, that acts as

$$
\mathcal{M}_{A}(\rho)=\sum_{i}\langle i|\rho| i\rangle|0\rangle\left\langle\left. 0\right|_{A} .\right.
$$

All classical-quantum channels are entanglement-breaking [13], however, the channel $\mathcal{M}_{A} \otimes \operatorname{id}_{B}$ is not $A$-unital, as we have $\left(\mathcal{M}_{A} \otimes \mathrm{id}_{B}\right)\left(\frac{I_{A}}{d} \otimes \sigma_{B}\right)=|0\rangle\langle 0| \otimes \sigma_{B}$ which is not of the form $\frac{I_{A}}{d} \otimes \gamma_{B}$ for some $\gamma_{B} \in \mathcal{D}(B)$.

$A$-unital channels are closely related to unital channels. Indeed, as given in Def. 5 , while $A$-unital channels map states of the form $\frac{I_{A}}{d_{A}} \otimes \sigma_{B}$ to $\frac{I_{A}}{d_{A}} \otimes \sigma_{B}^{\prime}$, unital channels map $\frac{I_{A B}}{d_{A B}}$ to $\frac{I_{A B}}{d_{A B}}$. In the next section, we explore this connection further. We show that while $A$-unital channels are the largest class of completely free channels for $\mathcal{F}^{c}(A \mid B)$, unital channels form the largest class of completely free channels for a previously defined, related set $\mathcal{F}^{a c}(A \mid B)$, the set of all states whose conditional entropy remains non-negative under global unitary operations. The relationship between $A$-unital, $B$-unital and unital channels is depicted in Fig. 4.

\section{Unital Operations and Absolute Non-Negative Conditional Entropy}

\subsection{Absolutely Non-Negative Conditional Entropy States}

Previously, the absolute version of $\mathcal{F}^{c}(A \mid B)$ (analogous to absolutely separable states [29, 30]) was defined and characterized for the case where $d_{A}=d_{B}=2$ [27]. Here, we extend the definition by characterizing it for the case where $d_{A}=d_{B}=d$.

Definition 9. A quantum state $\rho \in \mathcal{D}(A B)$, where $d_{A}=d_{B}=d$, which satisfies $S_{A \mid B}\left(U \rho U^{\dagger}\right) \geq 0$ for all unitary channels $U: \mathcal{D}(A B) \rightarrow \mathcal{D}(A B)$ is called an absolutely non-negative conditional entropy state. The set of absolutely non-negative conditional entropy states in $\mathcal{D}(A B)$ is denoted by $\mathcal{F}^{a c}(A \mid B)$.

The set $\mathcal{F}^{a c}(A \mid B)$ is well-characterized in terms of the von Neumann entropy, as shown in the following theorem. 


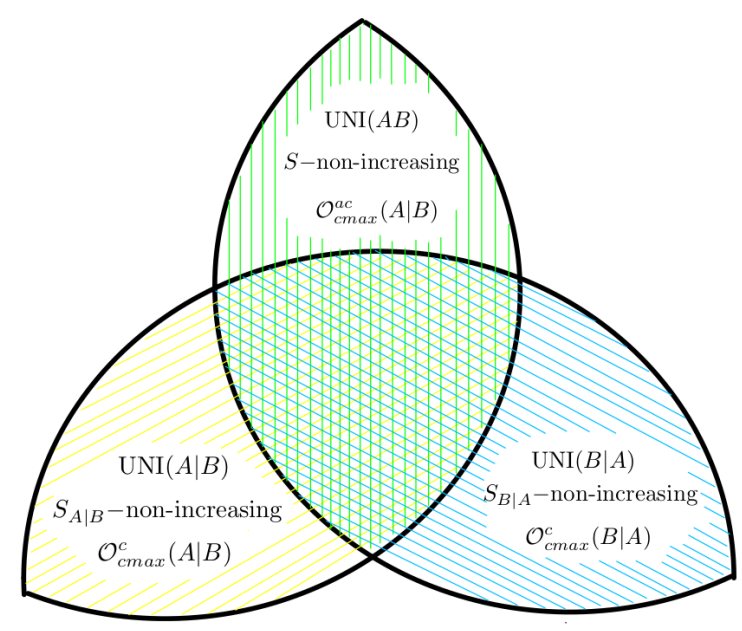

Figure 4: The relationship between $A$-unital, $B$-unital and unital channels when $d_{A}=d_{B}$.

Theorem 4. $\rho_{A B} \in \mathcal{F}^{a c}(A \mid B)$ if and only if $S\left(\rho_{A B}\right) \geq \log d$.

Proof. Let $\rho_{A B} \in \mathcal{D}(A B)$ and $S\left(\rho_{A B}\right) \geq \log d$. As the maximum von Neumann entropy of the subsystem $B$ is upperbounded by $\log d$, we have $\forall U, S\left(U \rho_{A B} U^{\dagger}\right)=S\left(\rho_{A B}\right) \geq$ $\log d \geq S\left(\left(U \rho_{A B} U^{\dagger}\right)_{B}\right)$. Therefore, $\forall U, S_{A \mid B}\left(U \rho U^{\dagger}\right)=S\left(U \rho U^{\dagger}\right)-S\left(\left(U \rho_{A B} \rho^{\dagger}\right)_{B}\right) \geq 0$ and $\rho_{A B} \in \mathcal{F}^{a c}(A \mid B)$.

To prove the "only if" part of the statement, consider the following set of vectors $\left\{\left|\psi_{n m}\right\rangle\right\}_{n m}$ which is a basis for the vector space $\mathcal{H}^{A} \otimes \mathcal{H}^{B}[31]$

$$
\left|\psi_{n m}\right\rangle=\sum_{j} e^{2 \pi i j n / d}|j\rangle \otimes|(j+m) \bmod d\rangle / \sqrt{d} .
$$

Notice that for each $\left|\psi_{n m}\right\rangle$, we have $\left(\left|\psi_{n m}\right\rangle\left\langle\psi_{n m}\right|\right)_{B}=\frac{I}{d}$. Now, let $\sigma_{A B} \in \mathcal{F}^{a c}(A \mid B)$. Let the set of vectors $\left\{\left|\gamma_{n m}\right\rangle\right\}_{n m}$ be the eigenbasis of $\sigma_{A B}$. Consider the unitary channel $U_{e} \equiv \sum_{n m}\left|\psi_{n m}\right\rangle\left\langle\gamma_{n m}\right|$. The state $U_{e} \sigma_{A B} U_{e}^{\dagger}$ is diagonal in the $\left|\psi_{n m}\right\rangle$ basis, hence, $\left(U_{e} \sigma_{A B} U_{e}^{\dagger}\right)_{B}=\frac{I}{d}$, and $S\left(\left(U_{e} \sigma_{A B} U_{e}^{\dagger}\right)_{B}\right)=\log d$.

By definition, we have $S_{A \mid B}\left(U \sigma_{A B} U^{\dagger}\right) \geq 0$ for all $U$, in particular for $U_{e}$. Therefore, $S_{A \mid B}\left(U_{e} \sigma_{A B} U_{e}^{\dagger}\right)=S\left(U_{e} \sigma_{A B} U_{e}^{\dagger}\right)-S\left(\left(U_{e} \sigma_{A B} U_{e}^{\dagger}\right)_{B}\right) \geq 0$, which implies that $S\left(\sigma_{A B}\right)$ $\log d \geq 0$. Thus, $\forall \sigma_{A B} \in \mathcal{F}^{a c}(A \mid B)$, we have $S\left(\sigma_{A B}\right) \geq \log d$.

\subsection{Completely Free Operations for Absolute Non-Negative Conditional Entropy States}

Here, we define the class of completely free operations for $\mathcal{F}^{a c}(A \mid B)$, respecting the $d_{A}=$ $d_{B}=d$ condition in the definition of $\mathcal{F}^{a c}(A \mid B)$.

Definition 10. A channel $\mathcal{N}_{A B}: \mathcal{D}(A B) \rightarrow \mathcal{D}(A B)$ is completely free for $\mathcal{F}^{a c}(A \mid B)$ if for all finite dimensional Hilbert spaces $\mathcal{H}^{A^{\prime}}$ and $\mathcal{H}^{B^{\prime}}$, where $d_{A^{\prime}}=d_{B^{\prime}}=d^{\prime}$ and $\rho \in \mathcal{F}^{a c}\left(A^{\prime} A \mid B^{\prime} B\right)$, we have $\left(\operatorname{id}_{A^{\prime} B^{\prime}} \otimes \mathcal{N}_{A B}\right)(\rho) \in \mathcal{F}^{a c}\left(A^{\prime} A \mid B^{\prime} B\right)$. The class of all such completely free operations mapping $\mathcal{D}(A B)$ to itself is denoted by $\mathcal{O}_{\text {cmax }}^{a c}(A \mid B)$.

We now prove that for the above definition of $\mathcal{O}_{c m a x}^{a c}(A \mid B)$, unitality is a necessary and sufficient condition for complete freeness. 


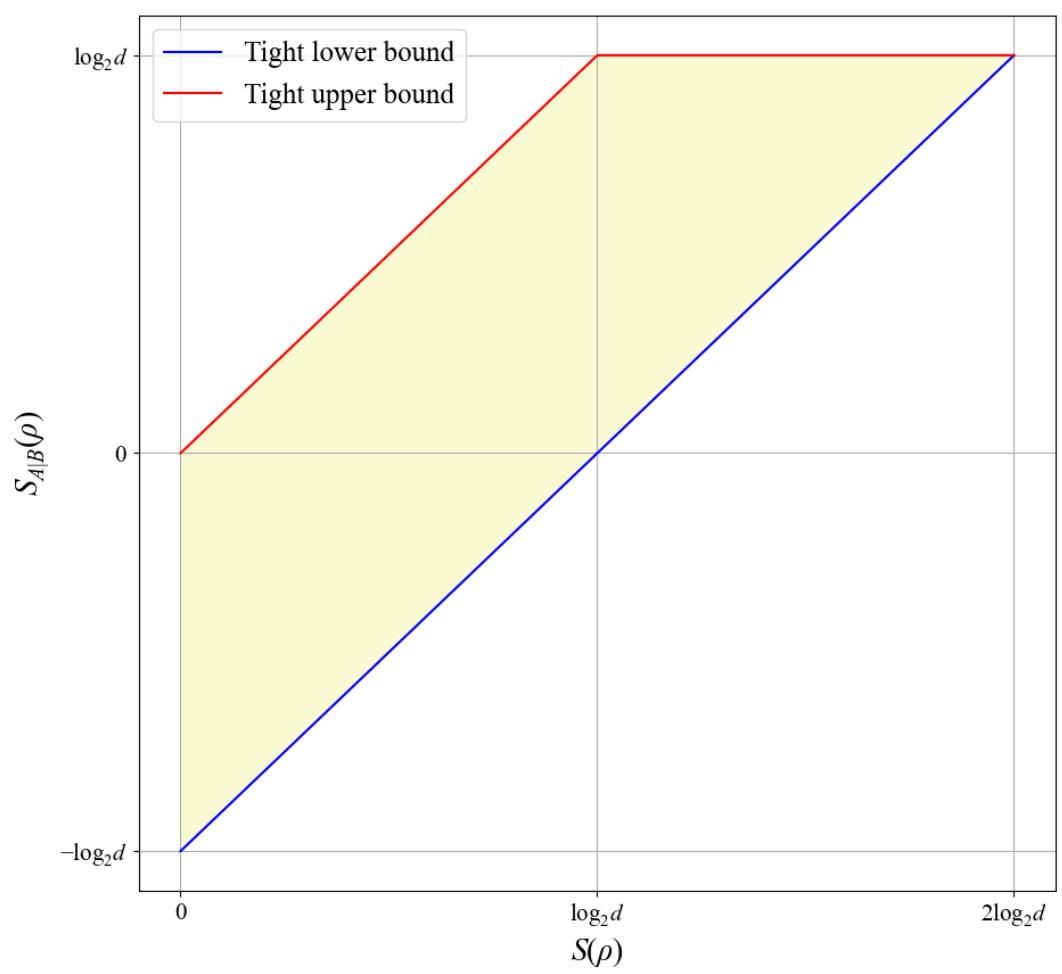

Figure 5: Upper and lower bounds for $S_{A \mid B}(\rho)$ given $S(\rho)$.

Theorem 5. A channel $\mathcal{N} \in \mathcal{O}_{\text {cmax }}^{a c}(A \mid B)$ if and only if it is unital.

Proof. Let $\mathcal{H}^{A}, \mathcal{H}^{B}, \mathcal{H}^{A^{\prime}}$ and $\mathcal{H}^{B^{\prime}}$ be finite dimensional Hilbert spaces such that $d_{A}=d_{B}=$ $d$ and $d_{A^{\prime}}=d_{B^{\prime}}=d^{\prime}$. Let $\mathcal{N}_{A B}$ be a unital channel mapping $\mathcal{D}(A B)$ to $\mathcal{D}(A B)$. Then, notice that the channel $\mathcal{N}^{\prime}=\operatorname{id}_{A^{\prime} B^{\prime}} \otimes \mathcal{N}_{A B}$ is also a unital channel mapping $\mathcal{D}\left(A^{\prime} A B^{\prime} B\right)$ to itself. This unitality implies we have $S\left(\mathcal{N}^{\prime}(\rho)\right) \geq S(\rho) \forall \rho \in \mathcal{D}\left(A^{\prime} A B^{\prime} B\right)$ [13]. Hence, $\forall \sigma \in \mathcal{F}^{a c}\left(A^{\prime} A \mid B^{\prime} B\right)$, we have $S\left(\mathcal{N}^{\prime}(\sigma)\right) \geq S(\sigma) \geq \log d d^{\prime}$, using Theorem 4. Thus, $\mathcal{N}^{\prime}(\sigma) \in \mathcal{F}^{a c}\left(A^{\prime} A \mid B^{\prime} B\right)$. This implies that $\mathcal{N}^{\prime}$ is a free operation, which in turn implies that $\mathcal{N}_{A B} \in \mathcal{O}_{\text {cmax }}^{a c}(A \mid B)$.

Let $\mathcal{M}_{A B}$ be a non-unital channel in $\mathcal{D}(A B)$. Hence, $\mathcal{M}_{A B}\left(\frac{I_{A B}}{d^{2}}\right)=\sigma_{A B}$ where $\sigma_{A B} \neq \frac{I_{A B}}{d^{2}}$. Thus, $S\left(\sigma_{A B}\right)<\log d^{2}$, since the maximum entropy for $\rho \in \mathcal{D}(A B)=$ $\log d^{2}$ and is achieved only at $\frac{I_{A B}}{d^{2}}$. Consider the state $\chi=\gamma_{A^{\prime} B^{\prime}} \otimes \frac{I_{A B}}{d^{2}}$ where $\gamma$ is a pure state and $d^{\prime}=d$. Hence $S(\chi)=\log d^{2}$ which implies that $\chi \in \mathcal{F}^{a c}\left(A^{\prime} A \mid B^{\prime} B\right)$. We have $\chi^{\prime}=\left(\operatorname{id}_{A^{\prime} B^{\prime}} \otimes \mathcal{M}_{A B}\right)(\chi)=\gamma_{A^{\prime} B^{\prime}} \otimes \sigma_{A B}$. Therefore, $S\left(\chi^{\prime}\right)<\log d^{2}$ and $\chi^{\prime} \notin$ $\mathcal{F}^{a c}\left(A^{\prime} A \mid B^{\prime} B\right)$. This implies that $\operatorname{id}_{A^{\prime} B^{\prime}} \otimes \mathcal{M}_{A B}$ is not a free operation, and therefore, $\mathcal{M}_{A B} \notin \mathcal{O}_{c \max }^{a c}(A \mid B)$. 


\section{Upper and Lower Bounds for Conditional Entropy given Entropy}

The characterization of $\mathcal{F}^{a c}(A \mid B)$ via entropy suggests that the entropy of a state can determine the values of conditional entropy that the state can possess. In the following section, we discuss upper and lower bounds for conditional entropy, given the entropy of a state $\rho_{A B}$, where $d_{A}=d_{B}=d$.

Theorem 6. Let $E_{x} \equiv\left\{S_{A \mid B}(\rho) \mid S(\rho)=x \forall \rho \in \mathcal{D}(A B)\right\}$. Then, the tight lower bound of $E_{x}$ is $x-\log d$ and the tight upper bound of $E_{x}$ is $\max (x, \log d)$.

Proof. Fig. 5 depicts the bounds of the above theorem. The lower bound is proven in Lemma 8 and the upper bound is proven in Lemmas 9 and 10.

Lemma 8. $\forall x \in[0,2 \log d]$, the tight lower bound of $E_{x} \equiv\left\{S_{A \mid B}(\rho) \mid S(\rho)=x \wedge \rho \in\right.$ $\mathcal{D}(A B)\}$ is $x-\log d$.

Proof. Consider $\rho \in \mathcal{D}(A B) \mid S(\rho)=x$. Since $S\left(\rho_{B}\right) \leq \log d$ (Theorem 11.8 in [28]), we have $S_{A \mid B}(\rho) \geq x-\log d$. Thus,

$$
\text { a lower bound of } E_{x} \text { is } x-\log d \text {. }
$$

It now remains to show that $\forall x \in[0,2 \log d], x-\log d \in E_{x}$. Consider the function $\mathcal{R}:[0,1] \rightarrow \mathcal{D}(A B): p \rightarrow p\left|\phi^{+}\right\rangle\left\langle\phi^{+}\right|+(1-p) \frac{I}{d^{2}}$ where $\left|\phi^{+}\right\rangle=\sum_{i=0}^{d-1} \frac{|i i\rangle}{\sqrt{d}}$. Note that $\forall p \in[0,1]$, we have $\mathcal{R}(p)_{B}=\frac{I}{d}$. Thus,

$$
S\left(\mathcal{R}(p)_{B}\right)=\log d .
$$

As $S: \mathcal{D}(A B) \rightarrow[0,2 \log d]$ is a continuous function (Box 11.2 in [28]) and $\mathcal{R}$ can be shown to be a continuous function ${ }^{2}$, we have $S \circ \mathcal{R}:[0,1] \rightarrow[0,2 \log d]$ is a continuous function. We have $S(\mathcal{R}(0))=2 \log d$ and $S(\mathcal{R}(1))=0$. Thus, by the intermediate value theorem,

$$
\forall x \in[0,2 \log d], \exists p_{x} \in[0,1] \mid S\left(\mathcal{R}\left(p_{x}\right)\right)=x .
$$

Using Eq. 30 and 31, we have

$$
S_{A \mid B}\left(\mathcal{R}\left(p_{x}\right)\right)=S\left(\mathcal{R}\left(p_{x}\right)\right)-S\left(\mathcal{R}\left(p_{x}\right)_{B}\right)=x-\log d .
$$

$S\left(\mathcal{R}\left(p_{x}\right)\right)=x$ (Eq. 31) implies that $S_{A \mid B}\left(\mathcal{R}\left(p_{x}\right)\right)=x-\log d \in E_{x}$ (Eq. 32). Thus, as $x-\log d$ is a lower bound of $E_{x}$, and $x-\log d \in E_{x}$, it is the tight lower bound.

Lemma 9. $\forall x \in[0, \log d]$, the tight upper bound of $E_{x} \equiv\left\{S_{A \mid B}(\rho) \mid S(\rho)=x \wedge \rho \in\right.$ $\mathcal{D}(A B)\}$ is $x$.

Proof. Consider $\rho \in \mathcal{D}(A B) \mid S(\rho)=x$. Since $S\left(\rho_{B}\right) \geq 0$, we have $S_{A \mid B}(\rho) \leq x$. Thus, an upper bound of $E_{x}$ is $x$. It now remains to show that $\forall x \in[0, \log d], x \in E_{x}$. For a given $x \in[0, \log d]$, consider states of the form $\sigma=\sigma_{A} \otimes \sigma_{B}$, where $S\left(\sigma_{A}\right)=x$ and $\sigma_{B}$ is any pure state. We have $S(\sigma)=x+0=x$, which implies $S_{A \mid B}(\sigma)=x-0=x \in E_{x}$.

Therefore, since $x$ is an upperbound of $E_{x}$ and $x \in E_{x}$, it is a tight upper bound.

Lemma 10. $\forall x \in[\log d, 2 \log d]$, the tight upper bound of $E_{x} \equiv\left\{S_{A \mid B}(\rho) \mid S(\rho)=x \wedge \rho \in\right.$ $\mathcal{D}(A B)\}$ is $\log d$.

\footnotetext{
${ }^{2}$ This can be shown using the $\epsilon-\delta$ definition of continuity.
} 
Proof. From Theorem 11.4.1 in [13] and Theorem 11.8 in [28], we have $\forall \rho \in$ $\mathcal{D}(A B), \quad S_{A \mid B}(\rho) \leq S\left(\rho_{A}\right) \leq \log d$. Therefore, $\log d$ is also an upper bound for $E_{x}$. It now remains to show that $\forall x \in[\log d, 2 \log d], \log d \in E_{x}$. Consider states of the form $\sigma=\frac{I}{d} \otimes \sigma_{B}$ where $S\left(\sigma_{B}\right)=x-\log d$. We have $S(\sigma)=x$ which implies that $S_{A \mid B}(\sigma)=\log d \in E_{x}$. Therefore, since $\log d$ is an upper bound for $E_{x}$ and $\log d \in E_{x}$, it is a tight upper bound.

Theorem 6 follows from the lower bound proved in Lemma 8 and the upper bounds proved in Lemmas 9 and 10.

\section{Conclusion}

In this paper, we introduce the class of $A$-unital channels, and show that this is equal to the largest class of completely free channels for the set of states with non-negative conditional entropy $\left(\mathcal{F}^{c}(A \mid B)\right)$. We also show that this is the largest class of channels that do not decrease the conditional entropy of a state. We discuss the relation between $A$-unital channels, separable channels and entanglement-breaking channels. Furthermore, we extended the characterization of the set of states that possess non-negative conditional entropy even under global unitary transformations $\left(\mathcal{F}^{a c}(A \mid B)\right)$ from the $2 \otimes 2$ case to the $d \otimes d$ case. We demonstrate that unital channels are the largest class of completely free operations for this set. Finally, we provide upper and lower bounds for the conditional entropy of a state as a function of the entropy of a state. We conclude with some questions for further research that emerge from our work.

1. Characterization of $\mathcal{F}^{a c}(A \mid B)$ for the $d_{A} \otimes d_{B}$ case. In this work, we show that when $d_{A}=d_{B}=d, \mathcal{F}^{a c}(A \mid B)$ is the set of states in $\mathcal{D}(A B)$ for which $S\left(\rho_{A B}\right) \geq \log d$. An interesting problem is the extension of this characterization to the case $d_{A} \neq d_{B}$. This is important for the full characterization of completely free operations for $\mathcal{F}^{a c}(A \mid B)$.

2. Decomposition of A-unital channels and a Choi matrix representation. A necessary and sufficient condition for the unitality of a channel is for it to be expressed as an affine combination of unitary quantum channels [32]. Providing a similar decomposition for A-unital channels is an open problem. Moreover, separable channels, completely PPT-preserving channels and entanglement-breaking channels all have elegant characterizations in terms of Choi matrices [4, 13, 33-36]. A similar characterization remains to be given for $A$-unital channels.

3. State conversion under A-unital channels. According to Uhlmann's theorem, a state $\rho$ can be converted to state $\sigma$ under unital channels if and only if $\sigma \prec \rho$, that is $\rho$ majorizes $\sigma[37,38]$. What are the conditions for state conversion under $A$-unital channels?

Note added: Also see related independent work by Brandsen et al. [39]

\section{Acknowledgements}

We gratefully acknowledge Prof. Arun K. Pati for his enriching discussions, and Mohammad Alhejji for his fruitful comments. 


\section{References}

[1] Ryszard Horodecki, Paweł Horodecki, Michał Horodecki, and Karol Horodecki. Quantum entanglement. Rev. Mod. Phys., 81:865-942, Jun 2009. DOI: 10.1103/RevModPhys.81.865. URL https://link.aps.org/doi/10.1103/RevModPhys.81.865.

[2] Eric Chitambar and Gilad Gour. Quantum resource theories. Rev. Mod. Phys., 91: 025001, Apr 2019. DOI: 10.1103/RevModPhys.91.025001. URL https://link.aps . org/doi/10.1103/RevModPhys.91.025001.

[3] Eric Chitambar, Debbie Leung, Laura Mančinska, Maris Ozols, and Andreas Winter. Everything you always wanted to know about LOCC (but were afraid to ask). Communications in Mathematical Physics, 328(1):303-326, March 2014. DOI: 10.1007/s00220-014-1953-9. URL https://doi.org/10.1007/s00220-014-1953-9.

[4] J. I. Cirac, W. Dür, B. Kraus, and M. Lewenstein. Entangling operations and their implementation using a small amount of entanglement. Phys. Rev. Lett., 86:544-547, Jan 2001. DOI: 10.1103/PhysRevLett.86.544. URL https://link.aps .org/doi/10. 1103/PhysRevLett.86.544.

[5] V. Vedral and M. B. Plenio. Entanglement measures and purification procedures. Phys. Rev. A, 57:1619-1633, Mar 1998. DOI: 10.1103/PhysRevA.57.1619. URL https://link.aps.org/doi/10.1103/PhysRevA.57.1619.

[6] Michael Horodecki, Peter W. Shor, and Mary Beth Ruskai. Entanglement breaking channels. Reviews in Mathematical Physics, 15(06):629-641, August 2003. DOI: 10.1142/s0129055x03001709. URL https://doi.org/10.1142/s0129055x03001709.

[7] C. Macchiavello and M. Rossi. Quantum channel detection. Phys. Rev. A, 88:042335, Oct 2013. DOI: 10.1103/PhysRevA.88.042335. URL https://link.aps.org/doi/ 10.1103/PhysRevA.88.042335.

[8] Colin Do-Yan Lee and John Watrous. Detecting mixed-unitary quantum channels is NP-hard. Quantum, 4:253, April 2020. DOI: 10.22331/q-2020-04-16-253. URL https://doi.org/10.22331/q-2020-04-16-253.

[9] Ashley Montanaro and Ronald de Wolf. A survey of quantum property testing. Theory of Computing, 1(1):1-81, 2016. DOI: 10.4086/toc.gs.2016.007. URL https://doi . org/10.4086/toc.gs.2016.007.

[10] N. Milazzo, D. Braun, and O. Giraud. Truncated moment sequences and a solution to the channel separability problem. Phys. Rev. A, 102:052406, Nov 2020. DOI: 10.1103/PhysRevA.102.052406. URL https://link.aps.org/doi/10.1103/ PhysRevA.102.052406.

[11] Sevag Gharibian. Strong np-hardness of the quantum separability problem. Quantum Info. Comput., 10(3):343-360, March 2010. ISSN 1533-7146. DOI: 10.26421/QIC10.34-11.

[12] N. J. Cerf and C. Adami. Negative entropy and information in quantum mechanics. Phys. Rev. Lett., 79:5194-5197, Dec 1997. DOI: 10.1103/PhysRevLett.79.5194. URL https://link.aps.org/doi/10.1103/PhysRevLett.79.5194.

[13] Mark M. Wilde. Quantum Information Theory. Cambridge University Press, 2016. DOI: 10.1017/9781316809976. URL https://doi.org/10.1017/9781316809976.

[14] Harold Ollivier and Wojciech H. Zurek. Quantum discord: A measure of the quantumness of correlations. Phys. Rev. Lett., 88:017901, Dec 2001. DOI: 10.1103/PhysRevLett.88.017901. URL https://link.aps.org/doi/10.1103/PhysRevLett.88. 017901.

[15] L Henderson and V Vedral. Classical, quantum and total correlations. Journal of Physics A: Mathematical and General, 34(35):6899-6905, Aug 2001. DOI: 

$2 \mathrm{~F} 34 \% 2 \mathrm{~F} 35 \% 2 \mathrm{~F} 315$.

[16] Chandrashekar Radhakrishnan, Mathieu Laurière, and Tim Byrnes. Multipartite generalization of quantum discord. Phys. Rev. Lett., 124:110401, Mar 2020. DOI: 10.1103/PhysRevLett.124.110401. URL https://link.aps.org/doi/ 10.1103/PhysRevLett.124.110401.

[17] Michał Horodecki, Jonathan Oppenheim, and Andreas Winter. Partial quantum information. Nature, 436(7051):673-676, August 2005. DOI: 10.1038/nature03909. URL https://doi.org/10.1038/nature03909.

[18] Michał Horodecki, Jonathan Oppenheim, and Andreas Winter. Quantum state merging and negative information. Communications in Mathematical Physics, 269(1):107136, October 2006. DOI: 10.1007/s00220-006-0118-x. URL https://doi.org/10. 1007/s00220-006-0118-x.

[19] Charles H. Bennett and Stephen J. Wiesner. Communication via one- and two-particle operators on einstein-podolsky-rosen states. Phys. Rev. Lett., 69:2881-2884, Nov 1992. DOI: 10.1103/PhysRevLett.69.2881. URL https://link.aps.org/doi/10. 1103/PhysRevLett.69.2881.

[20] D. Bruß, G. M. D'Ariano, M. Lewenstein, C. Macchiavello, A. Sen(De), and U. Sen. Distributed quantum dense coding. Phys. Rev. Lett., 93:210501, Nov 2004. DOI: 10.1103/PhysRevLett.93.210501. URL https://link.aps.org/doi/10.1103/ PhysRevLett.93.210501.

[21] R. Prabhu, Arun Kumar Pati, Aditi Sen(De), and Ujjwal Sen. Exclusion principle for quantum dense coding. Phys. Rev. A, 87:052319, May 2013. DOI: 10.1103/PhysRevA.87.052319. URL https://link.aps .org/doi/10.1103/PhysRevA .87.052319.

[22] Igor Devetak and Andreas Winter. Distillation of secret key and entanglement from quantum states. Proceedings of the Royal Society A: Mathematical, Physical and Engineering Sciences, 461(2053):207-235, January 2005. DOI: 10.1098/rspa.2004.1372. URL https://doi.org/10.1098/rspa.2004.1372.

[23] Dong Yang, Karol Horodecki, and Andreas Winter. Distributed private randomness distillation. Phys. Rev. Lett., 123:170501, Oct 2019. DOI: 10.1103/PhysRevLett.123.170501. URL https://link.aps.org/doi/10.1103/PhysRevLett. 123. 170501.

[24] Mario Berta, Matthias Christandl, Roger Colbeck, Joseph M. Renes, and Renato Renner. The uncertainty principle in the presence of quantum memory. Nature Physics, 6 (9):659-662, July 2010. DOI: 10.1038/nphys1734. URL https://doi.org/10.1038/ nphys 1734.

[25] Mahathi Vempati, Nirman Ganguly, Indranil Chakrabarty, and Arun K. Pati. Witnessing negative conditional entropy. Phys. Rev. A, 104:012417, Jul 2021. DOI: 10.1103/PhysRevA.104.012417. URL https://link.aps.org/doi/10.1103/ PhysRevA.104.012417.

[26] Nicolai Friis, Sridhar Bulusu, and Reinhold A Bertlmann. Geometry of two-qubit states with negative conditional entropy. Journal of Physics A: Mathematical and Theoretical, 50(12):125301, feb 2017. DOI: 10.1088/1751-8121/aa5dfd. URL https: //doi.org/10.1088/1751-8121/aa5dfd.

[27] Subhasree Patro, Indranil Chakrabarty, and Nirman Ganguly. Non-negativity of conditional von neumann entropy and global unitary operations. Phys. Rev. A, 96:062102, Dec 2017. DOI: 10.1103/PhysRevA.96.062102. URL https://link.aps.org/doi/ 10.1103/PhysRevA.96.062102. 
[28] Michael A. Nielsen and Isaac L. Chuang. Quantum Computation and Quantum Information. Cambridge University Press, 2009. DOI: 10.1017/cbo9780511976667. URL https://doi.org/10.1017/cbo9780511976667.

[29] Marek Kuś and Karol Życzkowski. Geometry of entangled states. Phys. Rev. A, 63: 032307, Feb 2001. DOI: 10.1103/PhysRevA.63.032307. URL https://link.aps. org/doi/10.1103/PhysRevA.63.032307.

[30] Saronath Halder, Shiladitya Mal, and Aditi Sen(De). Characterizing the boundary of the set of absolutely separable states and their generation via noisy environments. Phys. Rev. A, 103:052431, May 2021. DOI: 10.1103/PhysRevA.103.052431. URL https://link.aps.org/doi/10.1103/PhysRevA.103.052431.

[31] Charles H. Bennett, Gilles Brassard, Claude Crépeau, Richard Jozsa, Asher Peres, and William K. Wootters. Teleporting an unknown quantum state via dual classical and einstein-podolsky-rosen channels. Phys. Rev. Lett., 70:1895-1899, Mar 1993. DOI: 10.1103/PhysRevLett.70.1895. URL https://link.aps.org/doi/10. 1103/PhysRevLett.70.1895.

[32] Christian B. Mendl and Michael M. Wolf. Unital quantum channels - convex structure and revivals of birkhoff's theorem. Communications in Mathematical Physics, 289(3): 1057-1086, May 2009. DOI: 10.1007/s00220-009-0824-2. URL https://doi .org/10. $1007 / \mathrm{s} 00220-009-0824-2$.

[33] E. M. Rains. Bound on distillable entanglement. Phys. Rev. A, 60:179-184, Jul 1999. DOI: 10.1103/PhysRevA.60.179. URL https://link.aps.org/doi/10.1103/ PhysRevA.60.179.

[34] E.M. Rains. A semidefinite program for distillable entanglement. IEEE Transactions on Information Theory, 47(7):2921-2933, 2001. DOI: 10.1109/18.959270.

[35] Bartosz Regula, Kun Fang, Xin Wang, and Mile Gu. One-shot entanglement distillation beyond local operations and classical communication. New Journal of Physics, 21(10):103017, October 2019. DOI: 10.1088/1367-2630/ab4732. URL https: //doi.org/10.1088/1367-2630/ab4732.

[36] Min Jiang, Shunlong Luo, and Shuangshuang Fu. Channel-state duality. Phys. Rev. A, 87:022310, Feb 2013. DOI: 10.1103/PhysRevA.87.022310. URL https://link. aps.org/doi/10.1103/PhysRevA.87.022310.

[37] Michael A. Nielsen. An introduction to majorization and its applications to quantum mechanics. 2002. URL https://michaelnielsen.org/blog/talks/2002/maj/ book.ps.

[38] Yuan Li and Paul Busch. Von neumann entropy and majorization. Journal of Mathematical Analysis and Applications, 408(1):384-393, December 2013. DOI: 10.1016/j.jmaa.2013.06.019. URL https://doi.org/10.1016/j.jmaa.2013. 06.019 .

[39] Sarah Brandsen, Isabelle J Geng, Mark M Wilde, and Gilad Gour. Quantum conditional entropy from information-theoretic principles. arXiv preprint arXiv:2110.15330, 2021. URL https://arxiv.org/abs/2110.15330. 